\title{
Long-Term Survivors of Metastatic Colorectal Cancer: A Tertiary Care Centre Experience
}

\author{
Aparna Sharma ${ }^{1}$ Atul Sharma ${ }^{1}$ Vinod Sharma ${ }^{1}$ Sunil Kumar ${ }^{2}$ Akash Kumar ${ }^{1}$ SVS Deo ${ }^{2}$ \\ Sushmita Pathy ${ }^{3}$ NK Shukla ${ }^{2}$ Raja Pramanik ${ }^{1}$ Vinod Raina ${ }^{1}$ Sanjay Thulkar ${ }^{4}$ Rakesh Kumar $^{5}$ \\ BK Mohanti ${ }^{3}$
}

1Department of Medical Oncology, Dr. B. R. Ambedkar Institute Rotary Cancer Hospital, All India Institute of Medical Sciences, New Delhi, India.

${ }^{2}$ Department of Surgical Oncology, Dr. B. R. Ambedkar Institute Rotary Cancer Hospital, All India Institute of Medical Sciences, New Delhi, India

${ }^{3}$ Department of Radiation Oncology, Dr. B. R. Ambedkar Institute Rotary Cancer Hospital, All India Institute of Medical Sciences,

New Delhi, India

${ }^{4}$ Department of Radiodiagnosis, Dr. B. R. Ambedkar Institute Rotary Cancer Hospital, All India Institute of Medical Sciences, New Delhi, India

${ }^{5}$ Department of Nuclear Medicine, All India Institute of Medical Sciences, New Delhi, India

South Asian J Cancer 2021;00:87-91.
Address for correspondence Atul Sharma, Academic qualifications; MD, DM, Pincode : 110029,Department of Medical Oncology, Dr. B. R. Ambedkar Institute Rotary Cancer Hospital, All India Institute of Medical Sciences, New Delhi, 110029 India (e-mail: atul1@hotmail.com).

\section{Abstract

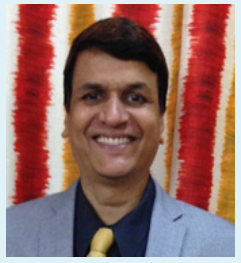 \\ Atul Sharma \\ Keywords \\ - metastatic colorectal cancer \\ - long-term survivors \\ - chemotherapy \\ - India}

Background Prognosis of metastatic colorectal cancer (mCRC) is poor and goal of treatment is mainly palliative unless there is limited metastatic disease which is surgically resectable. Here, we report a case series of long-term survivors treated predominantly with chemotherapy.

Methods This is a single-center retrospective analysis of patients of mCRC. Records of metastatic colorectal cancer patients registered at Institute Rotary Cancer Hospital, All India Institute of Medical Sciences, New Delhi, between the year 2005 and 2015 were retrieved and reviewed. Inclusion criteria were patients who survived 5 years or more, treated mainly by chemotherapy, with either initial presentation as metastatic disease or those who progressed after initial surgery with or without adjuvant therapy. The details about the patient characteristics, treatment, and outcome were collected. The data were censored on September 30, 2020.

Results Records of 370 mCRC patients were reviewed. Thirty-one patients with all the available details fulfilled the criteria for inclusion in the study. Median age was 53 years (range, 22-74 years). Sixteen were women (51.6\%). Twenty-four (77\%) were newly diagnosed cases with initial presentation as metastatic disease. Commonest site of primary was on the left $(21,67.6 \%)$ followed by right side and transverse colon in 5 patients each. Liver was the most common site of metastasis ( $n=18,58.06 \%$ ).
How to cite this article: S. Aparna, S. Atul, S. Vinod, K. Sunil, K. Akash, D. SVS, P. Sushmita, NK. Shukla, P. Raja, R. Vinod, T. Sanjay, K. Rakesh, BK. Mohanti. Long-Term Survivors of Metastatic Colorectal Cancer: A Tertiary Care Centre Experience. South Asian J Cancer 2021;10(2):87-91. (c) 2021. MedIntel Services Pvt Ltd.

This is an open access article published by Thieme under the terms of the Creative Commons Attribution-NonDerivative-NonCommercial-License, permitting copying and reproduction so long as the original work is given appropriate credit. Contents may not be used for commercial purposes, or adapted, remixed, transformed or built upon. (https://creativecommons.org/licenses/by-nc-nd/4.0/).

Thieme Medical and Scientific Publishers Private Ltd A-12, Second Floor, Sector -2, NOIDA -201301, India 
In metastatic setting, the most common chemotherapy regimen used in the first line was CAPOX ( $n=11,35.48 \%$ ). Only three patients could undergo metastatectomy. Monoclonal antibodies could be used only in 14 patients. Median overall survival (OS) of this cohort is 81.6 months (95\% confidence interval [CI], 69.73-117.9).

Conclusion A small but significant proportion of mCRC patients may achieve and maintain durable responses and long term survival with use of combination of chemotherapy with or without biologics.

\section{Introduction}

As per Globocan 2018 data colorectal cancer (CRC) is the third most common cancer (10.2\%) and the second most common cause of cancer-related death among all cancer cases. ${ }^{1}$ Metastatic colorectal cancer (mCRC) has dismal outcomes, with the 5 -year survival rate of less than $10 \%$. The median survival time of patients with untreated mCRC is approximately 5 months. ${ }^{2}$ The goal of treatment in majority of cases remain palliative unless there is limited metastatic disease which is resectable, as surgery is the only curative treatment option. ${ }^{3}$ However, the landscape of treatment of mCRC has significantly evolved over the past two decades with the availability of newer chemotherapeutic, targeted agents. ${ }^{4} \mathrm{~A}$ better understanding of the administration of combination chemotherapy (oxaliplatin and irinotecan) with use of biological agents (bevacizumab, cetuximab, and panitumumab), molecular biology of the disease, approval of newer agents (regorafenib, Her2neu inhibitor) immune checkpoint inhibitors, and improvement in supportive care delivery have largely revolutionized the outcomes of metastatic patients. ${ }^{5} \mathrm{~A}$ small subset of these patients may have durable responses and survival even without surgery. The data regarding this subset are lacking from the Indian as well as South East Asian subcontinent regarding long-term survivors. Here we report a case series from India, of long-term survivors of mCRC mainly treated with chemotherapy.

\section{Methods}

This is a single-center retrospective analysis of patients of mCRC registered at Institute Rotary Cancer Hospital, All India Institute of Medical Sciences, New Delhi, from the year 2005 to 2015. Main inclusion criteria were mCRC patients who were treated at the centre mainly with chemotherapy, who had either initial presentation as metastatic disease or those who progressed after initial surgery with or without adjuvant therapy, and those who survived for 5 or more years. Data were retrieved from the hospital-based record system. Demographic details including age, sex, Eastern cooperative oncology group (ECOG)performance status, primary and metastatic sites stage, and various treatment lines including biologics received by the patient were documented in a structured proforma. Treatment response and current status of the patient were obtained telephonically and from case records. Common terminology criteria for adverse events (CTCAE) 4.0 was used to document toxicities. Median and range were used to describe quantitative variables. Kaplan-Meier curve was used for survival analyses. Cox proportional hazards model was used to assess the association of clinical variables with survival. $P$ values of $<0.05$ were considered statistically significant. Statistical analysis was done using STATA software (ver13 StataCorp, College Station, Texas, United States). Data were censored on September 30, 2020.

\section{Results}

\section{Patient Characteristics}

In the duration extending from year 2005 to 2015, records of 370 mCRC patients were identified and reviewed. Thirty-eight patients fulfilled the criteria of survival beyond 5 years. However, details of treatment, evaluable response, and complete records were available for 31 patients. Median age was 53 years (range, 22-74 years). Sixteen patients were women (51.6\%). Out of the 31 patients, 13 (41.9\%) patients had comorbidities like diabetes, hypertension, or hypothyroidism. Twenty-eight (90.3\%) patients had ECOG PS 1 and two (6.4\%) patients presented with PS 2. Twenty-one (67.7\%) patients had left-sided colon cancer, whereas 5 patients (16.1\%) had right-sided colon cancer. Five (16.1\%) patients had involvement of transverse colon. The commonest histopathology grade was moderately differentiated adenocarcinoma (87\%); only one patient had mucinous histology. Liver was the most common predominant site of metastasis $(n=18,58 \%)$, followed by peritoneum ( $n=6,19.3 \%)$, and lung $(n=4,12.9 \%)$. Multiple unresectable liver metastases (defined as $>3$ ) were seen in 6 patients. Two patients had family history of gastrointestinal (GI) cancers without further details. Baseline median CEA (carcino embryonic antigen) was $6 \mathrm{ng} / \mathrm{mL}$ (range 2-900). RAS status was not available in all cases. K RAS status was available for 15 patients NRAS available in 3 patients and BRAF in one. Eleven patients were RAS wild type whereas one patient was documented as RAS mutated ( - Table1).

\section{Treatment Characteristics}

Twenty-four (77\%) of the patients presented as upfront metastatic disease. Seven (22.5\%) progressed as metastatic disease following previous curative resection with or without chemotherapy and radiotherapy. Median time to relapse was 484 days. Three patients were stage II and did not receive any 
Table 1 Patient characteristics

\begin{tabular}{|c|c|}
\hline Characteristics & $N=31(N) \%$ \\
\hline Median age & $53(22-74)$ \\
\hline \multicolumn{2}{|l|}{ Sex } \\
\hline Male & $15(48.39)$ \\
\hline Female & $16(51.61)$ \\
\hline \multicolumn{2}{|l|}{ ECOG PS } \\
\hline 0 & $1(3.23)$ \\
\hline 1 & $28(90.32)$ \\
\hline 2 & $2(6.45)$ \\
\hline \multicolumn{2}{|l|}{ Sidedness } \\
\hline Right & $5(16.13)$ \\
\hline Left & $21(67.74)$ \\
\hline Transverse colon & $5(16.13)$ \\
\hline \multicolumn{2}{|l|}{ Mutation status available } \\
\hline K RAS & $15(48.38)$ \\
\hline NRAS & $3(9.67)$ \\
\hline BRAF & $1(3.2)$ \\
\hline \multicolumn{2}{|l|}{ No. of previous lines received } \\
\hline 1 & $14(45.16)$ \\
\hline 2 & $8(25.81)$ \\
\hline 3 & $3(9.68)$ \\
\hline 4 & $1(3.23)$ \\
\hline 5 & $5(16.13)$ \\
\hline \multicolumn{2}{|l|}{ No. of metastatic sites } \\
\hline 1 & $27(87.1)$ \\
\hline 2 & $4(12.9)$ \\
\hline \multicolumn{2}{|l|}{ Site of metastasis } \\
\hline Liver & $18(58.06)$ \\
\hline Lung & 4 (12.9) \\
\hline Peritoneum & $6(19.35)$ \\
\hline Distant nodal & $5(16.13)$ \\
\hline \multicolumn{2}{|c|}{$\begin{array}{l}\text { Biochemical parameters mean } \\
\text { (range) }\end{array}$} \\
\hline CEA levels (ng/dL) & $6(2-900)$ \\
\hline Hemoglobin (g/dL) & $11.7(6-16.4)$ \\
\hline $\begin{array}{l}\text { Total leucocyte count } \\
\left(10^{9} / \mathrm{L}\right)\end{array}$ & $7,600(4,500-15,500)$ \\
\hline Platelets $\left(10^{9} / \mathrm{L}\right)$ & $2.47(1.01-6.09)$ \\
\hline Serum albumin (g/dL) & $3.9(2.7-5.3)$ \\
\hline
\end{tabular}

adjuvant therapy. Remaining three patients received modified folinic acid, oxaliplatin, fluorouracil-based combination (FOLFOX) $(n=3)$ and one patient (rectal cancer) received adjuvant Chemoradiation. Twenty patients (64.5) received oxaliplatin-based first-line therapy. The most common regimen used as first line in palliative metastatic setting was capecitabine and oxaliplatin (CAPOX) ( $n=11,35.48 \%$ ) followed by $\operatorname{mFOLFOX~}(n=9,29.03 \%)$ and folinic acid, irinotecan, fluorouracil-based combination (FOLFIRI) ( $n=5,16.13 \%$ ). Only three patients underwent surgery for metastatic disease (pulmonary metastetectomy and hepatic resection.). The number of lines of chemotherapy given in metastatic setting were one ( $n=14,45.16 \%)$, two $(n=8,25.81 \%)$, three ( $n=3,9.68 \%)$, four $(n=1,3.23 \%)$, and five ( $n=5,13 \%)$. Total of 14 patients received monoclonal antibodies of which 11 patients received in first line, 10 in second line, and 5 in third line setting. The most common biologic agent used was cetuximab $(n=12)$ followed by bevacizumab ( $n=1$ ) and panitumumab (only 1 ). Three patients received both anti-epidermal growth factor receptor (EGFR) and anti-vascular endothelial growth factor (VEGF) targeting biological therapies.

\section{Adverse Events}

The most common adverse event profile CTCAE) grades 3 and 4 from long-term exposure to multiple lines of chemotherapy were hematological toxicity $(n=8)$ and peripheral neuropathy $(n=2)$. Two patients had grades 3 and 4 diarrhea and febrile neutropenia. Other less common grades 3 and 4 adverse events included rash and hand foot syndrome $(n=1)$ patient. No death was reported due to toxicity.

\section{Outcomes}

At the time of analysis, 10 patients are currently surviving. Six patients are maintaining radiological complete response. Three patients were lost to follow-up. Median overall survival (OS) of this cohort is 81.6 months (95\% confidence interval [CI], 69.73-117.9) (-Fig. 1) and median progression-free survival (PFS) to first line therapy was 17.23 months (95\% CI 13-46.8).

\section{Factors Predicting Survival}

We performed regression analysis to determine the factors that predicted survival. The variables considered in our study were age, sex, sidedness, number of chemotherapy lines received, upfront metastatic presentation, and use of biologic agents. Female gender was the only factor found significant ( $p=0.031$ ) in univariate analysis; however, the significance was not seen on multivariate regression analysis (- Table 2).

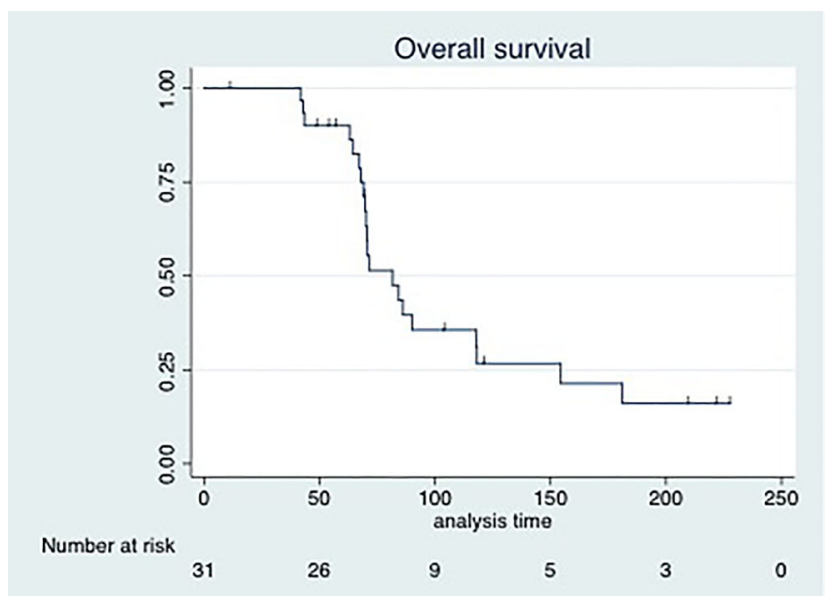

Fig. 1 Overall survival of metastatic colorectal cancers (long term survivors). 
Table 2 Treatment characteristics

\begin{tabular}{|l|l|}
\hline Characteristics & $(N) \%$ \\
\hline $\begin{array}{l}\text { Chemotherapy regimens in } \\
\text { first line }\end{array}$ & \\
\hline FOLFOX & $9(29.03)$ \\
\hline FOLFIRI & $5(16.13)$ \\
\hline Cap-OX & $11(35.48)$ \\
\hline Cap IRI & $2(6.45)$ \\
\hline IFL & $4(12.9)$ \\
\hline Biological agents received & $14(45.16)$ \\
\hline Yes & $17(54.8)$ \\
\hline No & 17.23 months(13.0-46.8) \\
\hline $\begin{array}{l}\text { Progression free survival } \\
\text { (first line) }\end{array}$ & 81.6 months(69.73-117.9) \\
\hline Median overall survival & \\
\hline
\end{tabular}

Table 3 Factors predicting overall survival

\begin{tabular}{|l|l|l|}
\hline Factors & $\begin{array}{l}\text { Univariate } \\
(p \text {-value })\end{array}$ & $\begin{array}{l}\text { Multivariate } \\
(p \text {-value })\end{array}$ \\
\hline $\begin{array}{l}\text { Age (57 or less vs. more } \\
\text { than 57) }\end{array}$ & 0.981 & - \\
\hline Sex & 0.031 & 0.174 \\
\hline Sidedness & 0.244 & - \\
\hline $\begin{array}{l}\text { Upfront metastatic } \\
\text { presentation }\end{array}$ & 0.48 & - \\
\hline $\begin{array}{l}\text { Number of lines used }(< \\
\text { or equal to 2) }\end{array}$ & 0.084 & 0.721 \\
\hline Use of biologics & 0.057 & 0.148 \\
\hline
\end{tabular}

\section{Discussion}

Long-term survivors of $\mathrm{mCRC}$ in literature has been defined conventionally as survival more than 5 years. $\mathrm{mCRC}$ has a median survival of 6 to 10 months with time-tested single agents like 5 FU. ${ }^{6}$ Advent of doublet chemotherapeutic regimen including oxaliplatin and irinotecan has further contributed to the armamentarium against this disease. ${ }^{7,8}$ The new era of biologicals and targeted therapy has ushered in changes in the outcomes and there is still uncertainty on how and to what extent these agents have an impact on survival. In the metastatic setting, "cure" per se has been a rare occurrence in colorectal cancers. However, in recent times long-term follow ups of various large multicenter trials have demonstrated a significant rise in patient population who have survived beyond the 5-year mark., ${ }^{9,10}$ Evolution in surgical techniques like hepatic resection of metastasis has also contributed to prolongation in survival as reported in various studies. ${ }^{11}$ Addressing the most common site of metastasis with improved infusional chemotherapy as well as newer surgical techniques has resulted in improvement of disease outcomes. ${ }^{12,13}$ Concrete data to substantiate correlations of factors to long-term survival and actual magnitude of patient population who are achieving cure in real life is not much reported. Till date, only two large studies,
MD Anderson Cancer Center ${ }^{9}$ and NCCTG, ${ }^{10}$ have addressed these patients and their characteristics. No data from India cohort are currently available which can provide us with the prevalence of prolonged survivors in our population.

In our study, $77 \%$ of patients presented to us as upfront metastatic disease. This analysis demonstrates that only a small proportion of our patients (10\%) underwent metastatectomy and majority received chemotherapy only as part of therapy. Hence, our analysis highlights good and durable response achieved with chemotherapy alone. Presently six patients are maintaining complete response. This is somewhat comparable to the large retrospective study of $\mathrm{MCRC}$ by Ferrarrato et al from MD Anderson Cancer Centre, ${ }^{9}$ where they reported six patients in CR on chemotherapy alone. Majority of our patients presented with left-sided colon cancer which now we know has a better prognosis. ${ }^{14}$ The most common used biologic in our study was cetuximab. However, as the current evidence of use of cetuximab according to sidedness of the colon cancer was not available at the time of institution of biologics in our patients, the response correlation according to primary tumor location needs to be assessed in a larger cohort. ${ }^{15}$

Toxicity is also a concern in long-term survivors and adverse effects like neuropathy can be disabling and have a bearing on quality of life. ${ }^{16}$ Seven percent of our patients had residual grade III peripheral neuropathy. ${ }^{17}$ Quality of life also takes precedence in analysis of long-term survivors and very few studies are available in literature to highlight this aspect. Our study did not include quality of life analysis.

It is hard to overlook the concepts of precision medicine and tailoring of therapy in subsequent lines. The approved agents like bevacizumab, cetuximab, and panitumumab have shown promise in prolongation of survival. ${ }^{18}$ Our study almost 50\% patients received at least one biologic agent. Genetic determinants of prolonged survival can be further analyzed in retrospective manner from bedside to the bench by tissue preservation and subjecting sample to specific of molecular and genetic testing.

The limitations of our study is its retrospective nature, less number of patients $(n=31)$, limited number of patients exposed to all available options, and unavailability of MSI (microsattelite instability) and RAS (RA|at sarcoma virus gene) status in majority of the patients.

Survival data on newer regimens are sparse owing to heterogeneous nature or retrospective nature of studies. Addition of biologics may have a contribution to patients achieving and maintaining complete and partial responses beyond 5 years and needs to be examined in detail.

\section{Conclusion}

The term "cure" might not be a remote possibility in mCRC. A close scrutiny needs to be maintained on all the large multicenter trials and their long-term follow-ups to ascertain and validate these data. Although the patient number is small, durable responses achieved with chemotherapy and biological agents alone in a metastatic setting is encouraging and possible. Analysis from large institutional database should be carried out. 
Funding

None.

Conflict of Interest

None declared.

\section{References}

1 Fact Sheets by Cancer. http://globocan.iarc.fr/Pages/fact_ sheets_cancer.aspx. Accessed April 27, 2018

2 Zacharakis M, Xynos ID, Lazaris A, et al. Predictors of survival in stage IV metastatic colorectal cancer. Anticancer Res 2010;30(2):653-660

3 Chow FC-L, Chok KS-H. Colorectal liver metastases: an update on multidisciplinary approach. World J Hepatol 2019;11(2):150-172

4 Kemeny NE. Treatment of metastatic colon cancer: "the times they are A-changing" J Clin Oncol 2013;31(16):1913-1916

5 Heinemann V, Douillard JY, Ducreux M, Peeters M. Targeted therapy in metastatic colorectal cancer-an example of personalised medicine in action. Cancer Treat Rev 2013;39(6):592-601

6 Perez N, Tournigand C, Mabro M, et al. Long term survival in metastatic colorectal cancer treated with leucovorin and 5-fluoro-uracil chemotherapy. Rev Med Interne 2004;25(2):124-128

7 Sanoff HK, Sargent DJ, Campbell ME, et al. Five-year data and prognostic factor analysis of oxaliplatin and irinotecan combinations for advanced colorectal cancer: N9741. J Clin Oncol 2008;26(35):5721-5727

8 Masi G, Loupakis F, Pollina L, et al. Long-term outcome of initially unresectable metastatic colorectal cancer patients treated with 5-fluorouracil/leucovorin, oxaliplatin, and irinotecan (FOLFOXIRI) followed by radical surgery of metastases. Ann Surg 2009;249(3):420-425
9 Ferrarotto R, Pathak P, Maru D, et al. Durable complete responses in metastatic colorectal cancer treated with chemotherapy alone. Clin Colorectal Cancer 2011;10(3):178-182

10 Dy GK, Hobday TJ, Nelson G, et al. Long-term survivors of metastatic colorectal cancer treated with systemic chemotherapy alone: a north central cancer treatment group review of 3811 patients, n0144. Clin Colorectal Cancer 2009;8(2):88-93

11 Kopetz S, Chang GJ, Overman MJ, et al. Improved survival in metastatic colorectal cancer is associated with adoption of hepatic resection and improved chemotherapy. J Clin Oncol 2009;27(22):3677-3683

12 Giacchetti S, Itzhaki M, Gruia G, et al. Long-term survival of patients with unresectable colorectal cancer liver metastases following infusional chemotherapy with 5-fluorouracil, leucovorin, oxaliplatin and surgery. Ann Oncol 1999;10(6):663-669

13 Dattatreya S. Metastatic colorectal cancer-prolonging overall survival with targeted therapies. South Asian J Cancer 2013;2(3):179-185

14 Baran B, Mert Ozupek N, Yerli Tetik N, Acar E, Bekcioglu O, Baskin Y. Difference between left-sided and right-sided colorectal cancer: a focused review of literature. Gastroenterol Res 2018;11(4):264-273

15 Holch JW, Ricard I, Stintzing S, Modest DP, Heinemann V. The relevance of primary tumour location in patients with metastatic colorectal cancer: a meta-analysis of first-line clinical trials. Eur J Cancer 2017;70:87-98

16 Van Loon K, Venook AP. Curable patient with metastatic colorectal cancer: balancing effective therapies and toxicities. J Clin Oncol 2014;32(10):991-996

17 Weickhardt A, Wells K, Messersmith W. Oxaliplatin-induced neuropathy in colorectal cancer [e-pub ahead of print]. J Oncol 2011;2011:201593

18 López-Gómez M, Merino M, Casado E. Long-term treatment of metastatic colorectal cancer with panitumumab. Clin Med Insights Oncol 2012;6:125-135Photo To Come 